\title{
Immunohistochemical and molecular analysis of Pasteurella multocida in a rabbit with suppurative pleuropneumonia
}

\author{
Kei UENOYAMA ${ }^{1) \#, ~ Y u i c h i ~ U E N O 2) \#, ~ K a o r i ~ T O S A K I ~}{ }^{3)}$, Yusuke ABETO4), \\ ${\text { Hirotaka } \text { ITO }^{5)} \text {, Ken KATSUDA }}^{2)}$ and Tomoyuki SHIBAHARA ${ }^{6,7) *}$ \\ 1)Pathological and Physiochemical Examination Division, Laboratory Department, Animal Quarantine Service, \\ 11-1 Haramachi, Isogo, Yokohama, Kanagawa 235-0008, Japan \\ ${ }^{2)}$ Division of Bacterial and Parasitic Disease, National Institute of Animal Health, National Agriculture and Food \\ Research Organization (NARO), 3-1-5 Kannondai, Tsukuba, Ibaraki 305-0856, Japan \\ 3)Tochigi Prefectural Kenou Livestock Hygiene Service Center, 6-8 Hiraidekogyodanchi, Utsunomiya, \\ Tochigi 321-0905, Japan \\ 4)Kagoshima Prefectural Kimotsuki Livestock Health and Hygiene Center,145-1 Nishiharaigawatyou, Kanoya, \\ Kagoshima 893-0025, Japan \\ ${ }^{5)}$ Hiroshima Prefectural Western Livestock Hygiene Service Center, 1-15 Saizyougozyoucho, Higashihiroshima, \\ Hiroshima 739-0013, Japan \\ ${ }^{6)}$ Division of Pathology and Pathophysiology, National Institute of Animal Health, National Agriculture and \\ Food Research Organization (NARO), 3-1-5 Kannondai, Tsukuba, Ibaraki 305-0856, Japan \\ ${ }^{7)}$ Department of Veterinary Science, Graduate School of Life and Environmental Sciences, Osaka Prefecture \\ University, 1-58 Rinku-oraikita, Izumisano, Osaka 598-8531, Japan
}

J. Vet. Med. Sci.

82(1): 89-93, 2020

doi: 10.1292/jvms.19-0561

Received: 11 October 2019

Accepted: 25 November 2019 Advanced Epub:

4 December 2019
ABSTRACT. A 1-month-old rabbit, imported as a pet by a distributor, died suddenly in the quarantine period in Japan due to suppurative pleuropneumonia. A bacterial isolate from its right lung was identified as Pasteurella multocida serotype A: 11 . The isolate was classified as ST204 using the RIRDC scheme of multilocus sequence typing, suggesting that the isolate was genetically related to European isolates of the same sequence type listed in the PubMLST database and not to four other isolates that originated from past imported rabbits. In the immunohistochemical assay, an antiserum recognizing the somatic serotype 11 antigen generated from chicken could specifically detect $P$. multocida, indicating that the antiserum for somatic serotyping was useful for immunohistochemical diagnosis of rabbit pasteurellosis.

KEY WORDS: immunohistochemical assay, multilocus sequence typing, Pasteurella multocida, rabbit, suppurative pleuropneumonia

Pasteurellosis is one of the most common diseases in rabbit and is prevalent in rabbit colonies worldwide [19]. Snuffles, pneumonia, otitis media, abscess formation, and conjunctivitis are the main pathological features of the disease [8, 9, 21], and have been reported previously [7, 17]. However, there is little information on immunohistochemical studies of the disease in rabbits, probably due to the difficulties in using antisera from rabbits with rabbit samples. Therefore, diagnosis of pasteurellosis in rabbits has been conducted only by histochemical techniques and bacterial identification.

An opportunistic pathogen, Pasteurella multocida, is the causative agent of pasteurellosis in rabbits. Rabbits can become infected with P. multocida immediately after birth, and the prevalence of colonization increases with age until 5 months [10]. Most adult rabbits are considered as carriers for this bacterium. Clinical isolates are divided into five capsular serotypes (A, B, D, E, and F) [2] and 16 somatic serotypes (1-16) based on the antigenicity of lipopolysaccharide [11]. Among them, isolates from rabbit have been classified into capsular serotypes A, D, and F, and Heddleston's somatic serotype 1, 3, 4, 12, and 15 [1, 3, 4, 11, 12, 15]. Capsular and somatic serotyping is conducted using antisera prepared from rabbit and chicken, respectively $[2,11]$.

Multilocus sequence typing (MLST), a method to characterize bacterial isolates by sequencing the internal fragments of housekeeping genes [5], has been used for the molecular epidemiological study of P. multocida [20]. According to a database of MLST, PubMLST (https://pubmlst.org/pmultocida/) [13], 92 isolates from rabbit had been grouped into 26 sequence types (STs), ST9, ST24, ST27, ST37, ST50, ST74, ST200, ST204, ST210, ST293-ST305, ST310-ST313, and ST365 by the RIRDC scheme

*Correspondence to: Shibahara, T.: tshiba@affrc.go.jp

\#These authors contributed equally to this work.

(O2020 The Japanese Society of Veterinary Science

This is an open-access article distributed under the terms of the Creative Commons Attribution Non-Commercial No Derivatives (by-nc-nd) License. (CC-BY-NC-ND 4.0: https://creativecommons.org/licenses/by-nc-nd/4.0/) 
of MLST as of September 2019. Among them, few P. multocida isolates from rabbit in Asia are registered in the database, and the genetic characteristics of Asian isolates from rabbit remain unclear.

This study describes a clinical case of pneumonic pasteurellosis observed in a rabbit imported from Taiwan to Japan. An immunohistochemical approach demonstrated pneumonic pasteurellosis in rabbit using antisera prepared from chicken for somatic serotyping of $P$. multocida. A molecular epidemiological study was also conducted using a $P$. multocida isolate from this study and from three other isolates from rabbits imported from China.

In 2017, one hundred and six rabbits (1-month-old) were reared in Taiwan. No rabbits were introduced into the flock in Taiwan for at least 30 days before shipment to Japan. The rabbits showed no evidence of any infectious diseases through careful clinical inspections conducted by the governmental authorities of Taiwan immediately before shipment to Japan. Upon delivery to Japan, none of the rabbits showed abnormal clinical signs or any evidence of disease. One rabbit died suddenly on the second day during the 3-day quarantine period in Japan, but no clinical abnormalities were observed in the remaining 105 rabbits.

Gross necropsy examination of the rabbit revealed suppurative pleuropneumonia. The right lung was covered with sheets of fibrin (Fig. 1a). Cut surfaces revealed multiple purulent lesions. The cranial lobe of the left lung was dark-red. No gross lesions were found in other organs, including the brain and spinal cord. At necropsy, tissue samples of the liver, spleen, kidney, heart, lungs, bladder, and brain were fixed in 10\% neutral-buffered formalin. Fixed tissues were embedded in paraffin wax, sectioned (3- $\mu \mathrm{m}$ thick) and stained for histological examination with hematoxylin and eosin (HE).

Histopathologically, multiple coagulative necrosis of the right lung was observed (Fig. 1b). The alveolar walls were necrotized with bacterial colonies. Neutrophils, macrophages, and bacterial cells were observed in the surrounding area of coagulative necrosis (Fig. 1c). The bacterial cells were gram-negative rod-shaped upon Gram staining and were surrounded by neutrophils and macrophages. Necrotic cells were observed in the bronchioles. Neutrophils, macrophages, and bacterial cells were observed in the pleura and the dark-red area of the left lung. The bacterial isolate (AQNT1704/1/NT1) from the lung was confirmed to be $P$. multocida somatic serotype 11 as described below.

Immunohistochemistry was performed to detect the specific antigen of $P$. multocida somatic serotype 11 . All formalin-fixed tissues were cut into $3-\mu \mathrm{m}$-thick sections, treated with $3 \%$ hydrogen peroxide in methanol (to suppress endogenous peroxidase activity), then treated with $0.1 \%$ actinase E solution (Kaken Pharmaceutical Co., Ltd., Tokyo, Japan) and incubated at $37^{\circ} \mathrm{C}$ for $20 \mathrm{~min}$ for antigen retrieval. The tissues were then incubated for $30 \mathrm{~min}$ at room temperature with anti P. multocida somatic serotype 11 antibody generated from chicken at 1:4,086 dilution. Then, the tissues were incubated with a secondary antibody (Biotinylated anti-chicken IgG $(\mathrm{H}+\mathrm{L})$ affinity purified BA-9010 Vector; San Diego, CA, USA) followed by peroxidase conjugated streptavidin (Histofine, Nichirei Bioscience Inc., Tokyo, Japan). After rinsing with phosphate buffered saline, the specimens were

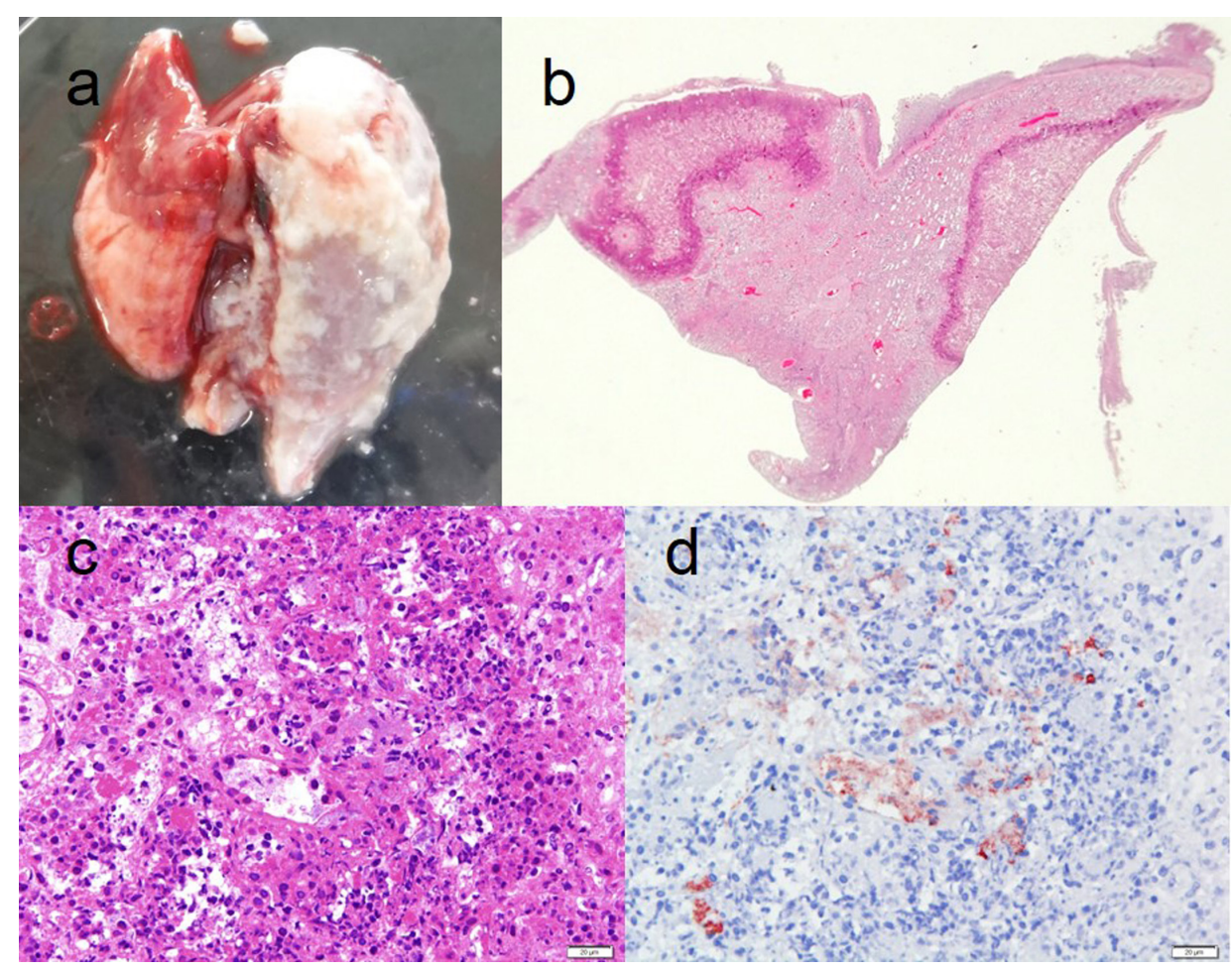

Fig. 1. a) Gross necropsy examination of the rabbit revealed suppurative pleuropneumonia. The right lung was covered with sheets of fibrin. b) Low magnification of the right lung characterized by the multiple coagulative necrosis. c) Neutrophils and macrophages were observed in the surrounding area of the coagulative necrosis. Bar $=20 \mu \mathrm{m}$. d) Immunohistochemical analysis demonstrated that the rod-shaped bacteria reacted with the chicken antibody against Pasteurella multocida serotype 11 . Bar=20 $\mu \mathrm{m}$. 
incubated with aminoethyl carbazole (Histofine Simple Stain AEC Solution, Nichirei Bioscience Inc., Tokyo, Japan) and substrate solution (Histofine Simple Stain AEC solution, Nichirei Bioscience Inc., Tokyo, Japan) at room temperature for 5 min, and then counterstained with hematoxylin. Simultaneously, hepatic tissues mechanically injected with $P$. multocida somatic serotype 11 (AQNT1704/1/NT1); serotypes A, B, D, E, and F; Mannheimia haemolytica serotypes A1, A2, A5-A9, A12-A14, and A16; Bibersteinia trehalosi serotypes T3, T4, T10, and T15; M. glucosida; M. varigena; Escherichia coli serotypes O45, O116, and O157; Salmonella enterica serovar Typhimurium; and S. enterica serovar Choleraesuis were used as positive and reference controls to verify the immunohistochemical specificity of the antiserum reaction. Negative controls were prepared by replacing the primary antibody with a commercial Tris-HCl buffer (antibody diluent with background reducing components; Dako, Tokyo, Japan).

Immunohistochemical analysis demonstrated that the rod-shaped bacteria reacted with the antibody against $P$. multocida serotype 11 (Fig. 1d). In addition, a strong positive reaction was detected only in the positive control sections of tissues containing P. multocida somatic serotype 11, but not in the other reference controls. Although a few and very weak cross-reaction was detected in the hepatic tissues mechanically injected with P. multocida serotypes B and E; M. haemolytica serotypes A5, A8, and A16; and B. trehalosi serotypes T4, they were easily distinguishable from that of tissues containing $P$. multocida somatic serotype 11 . Therefore, the anti $P$. multocida somatic serotype 11 antibody generated from chicken specifically reacted with $P$. multocida somatic serotype 11. This is the first report with anti $P$. multocida somatic chicken antisera that has proved useful for immunochemical identification. We found that the use of an antiserum generated from chicken against rabbits infected with P. multocida did not show nonspecific reactions to the rabbit tissues. In the immunohistochemical assay, an antiserum made from chicken for somatic serotype 11 could specifically detect the P. multocida antigen, showing that the antiserum for somatic serotyping was useful for immunochemical diagnosis in rabbits.

For bacterial culture, tissue samples of the liver, spleen, kidney, heart, lungs, bladder, and brain were stamped and inoculated on normal blood agar, deoxycholate-hydrogen sulfide-lactose (DHL) agar, and Gifu anaerobic medium (GAM) blood agar, and were then incubated at $37^{\circ} \mathrm{C}$ with $5 \% \mathrm{CO}_{2}$. Small mucoid colonies with no hemolysis were formed by plating the tissue samples of lungs after a 24-hr incubation and gram-negative coccobacilli were observed. The isolate from the right lung designated as AQNT1704/1/ NT1 was suspended in $20 \%$ glycerol containing brain heart infusion broth, and stored at $-80^{\circ} \mathrm{C}$ until use. No other bacterial colonies were grown from the lung sample and no bacteria were isolated from any of the other tissue samples. Both catalase (Kanto Chemical Co., Inc., Tokyo, Japan) and oxidase (Cytochrome Oxidase Test Strip Nissui, Tokyo, Japan) reactivities were confirmed to be positive.

To identify the isolate, AQNT1704/1/NT1, a biochemical assay and sequence analysis of 16S ribosomal RNA gene (16S rDNA) were carried out in this study. The biochemical assay was conducted using a commercial biochemical identification kit, API NE (BioMérieux, Lyon, France), and 16S rDNA sequencing was conducted according to a previous study [24]. Bacterial DNA was extracted from bacterial colonies using a DNA extraction kit (InstaGene Matrix; Bio-Rad Laboratories, Hercules, CA, USA) according to the manufacturer's instruction. A variable region of the 16S rDNA was amplified and sequenced using a MicroSeq 500 16S rDNA PCR/Sequencing Kit (Applied Biosystems Life Technologies, Carlsbad, CA, USA) [22]. The sequence data were collated into a database, EzBioCloud (https://www.ezbiocloud.net/) [25]. The result of API NE showed that AQNT1704/1/NT1 matched P. multocida (99.8\% good identification), and that of sequencing also showed the highest similarity (100\%) between strain AQNT1704/1/NT1 and the type strain of P. multocida, ATCC 43137. Three other P. multocida strains isolated from rabbits imported from China within the past quarantine periods, AQNT1405/1/NT1, AQNT1405/1/NT2, and AQNT1405/1/NT3 were also used in this study for epidemiological analysis of Asian isolates. These three strains were previously identified as $P$. multocida by the same biochemical assay and $16 \mathrm{~S}$ rDNA sequencing.

To estimate the capsular serotype of the four strains, multiplex capsular PCR typing targeting the cap loci was conducted according to a previous study [23]. DNA templates for PCR were prepared by boiling a 500- $\mu l$ suspension of 2-3 cultured colonies for $7 \mathrm{~min}$. After centrifugation, the supernatant was used as the DNA template. PCR products were fractionated by $1 \%$ agarose gel electrophoresis at $100 \mathrm{~V}$ for $30 \mathrm{~min}$. Gels were stained with $0.5 \mu \mathrm{g} / \mathrm{m} l$ ethidium bromide solution and photographed under ultraviolet light. Further, Heddleston's somatic serotyping using agar gel immunodiffusion (AGID) [11] was conducted for somatic characterization as well as to select the appropriate type of somatic antisera among the 16 serotypes for immunohistochemical analysis. Overnight cultures of the four strains on 5\% sheep blood containing BHI agar (Difco; BD, Franklin Lakes, NJ, USA) supplemented with $0.5 \%$ yeast extract (Difco) were suspended in PBS and incubated at $100^{\circ} \mathrm{C}$ for $1 \mathrm{hr}$. After centrifugation, the supernatant was used as the somatic antigen. Sixteen types of somatic antisera generated from chicken according to previous study [11] were used as antibodies. Each of the antisera for somatic serotyping used in this study were confirmed to not react with the strains of other somatic serotype antigens in AGID assay in advance. The agar gel for AGID was composed of $0.9 \%$ noble agar (Difco; BD, Franklin Lakes, NJ, USA); $8.5 \% \mathrm{NaCl}$ was prepared on a transparent plate and six wells of 4 mm diameter were cut equidistant around a central well. Thirty microliters of antiserum was added to the central well and somatic antigens were added to surrounding wells. Agar gels were incubated at $37^{\circ} \mathrm{C}$ for $24 \mathrm{hr}$. The somatic serotype was defined when an intense precipitate line was observed near the surrounding well. The strain AQNT1704/1/NT1 was thus determined to be capsular type A and somatic serotype 11 (A: 11). The serotypes of other strains were different from that of AQNT1704/1/NT1, in that, AQNT1405/1/NT1 and AQNT1405/1/NT2 were A: 3, and AQNT1405/1/NT3 was A: 3, 10, 12.

For the molecular epidemiological study, MLST was conducted on the four strains according to the protocol of the $P$. multocida RIRDC scheme [20] targeting seven housekeeping genes ( $a d k$, est, gdh, $m d h, p g i, p m i$, and $z w f)$. PCR products were treated with ExoSAP-IT (Affimetrix USB, Cleveland, OH, USA) according to the manufacturer's protocol to remove the excess nucleotides and primers. DNA sequencing was performed at our own facility using a BigDye Terminator Cycle Sequencing Kit v3.1 (Applied 
Biosystems, Foster City, CA, USA) on a 3130xl Genetic Analyzer apparatus (Applied Biosystems). The resulting sequences were edited using Sequencher ver. 4.8 (Gene Codes, Ann Arbor, MI, USA) software. The sequences were applied to the $P$. multocida MLST database and the appropriate ST was assigned. To estimate the genetic relationships among rabbit strains, a neighbor-joining phylogenetic tree [18] was generated based on the concatenated housekeeping gene sequences of rabbit isolates obtained in this study and from the database (Fig. 2) using MEGA7 (Molecular Evolutionary Genetics Analysis software, ver. 7.0) [14]. The tree was drawn to scale, with branch lengths in the same units as those of the evolutionary distances used to infer the phylogenetic tree. The evolutionary distances were computed using the p-distance method [16]. Sequence information of three $P$. multocida reference strains (ATCC 43137: ST13: the type strain of P. multocida subsp. multocida, NCTC 10204: ST14: the type strain of $P$. multocida subsp. gallicida, and NCTC 11995: ST15: the type strain of $P$. multocida subsp. septica) are also added in Fig. 2.

The genotype of strain AQNT1704/1/NT1 was determined to be ST204. According to the MLST database of P. multocida RIRDC scheme, four other rabbit strains isolated in Italy, Denmark, and Germany were classified into the same ST, indicating that these strains are genetically related. STs of the other isolates were different from that of AQNT1704/1/NT1; that is, AQNT1405/1/ NT1 and AQNT1405/1/NT2 are ST346, and AQNT1405/1/NT3 is ST50, which indicates that the origins of strains AQNT1405/1/ NT1 and AQNT1405/1/NT2, AQNT1405/1/NT3, and AQNT1704/1/NT1 are different, respectively (Fig. 2). This is the first report describing the isolation of ST346 strains, and the ST was found to be slightly aloof from other STs of rabbit isolates on the phylogenic tree (Fig. 2). On the contrary, ST50 strains have been isolated in multiple countries (Italy, Czech Republic, Spain, India, UK, Germany, Denmark, and China) and from multiple hosts other than rabbit (bovine, porcine, and avian).

In this study, we added the MLST information of four $P$. multocida isolates from Asia. However, the epidemiological value of the MLST data of rabbit isolates is still limited due to the low isolate numbers and the geographical bias of rabbit isolates in

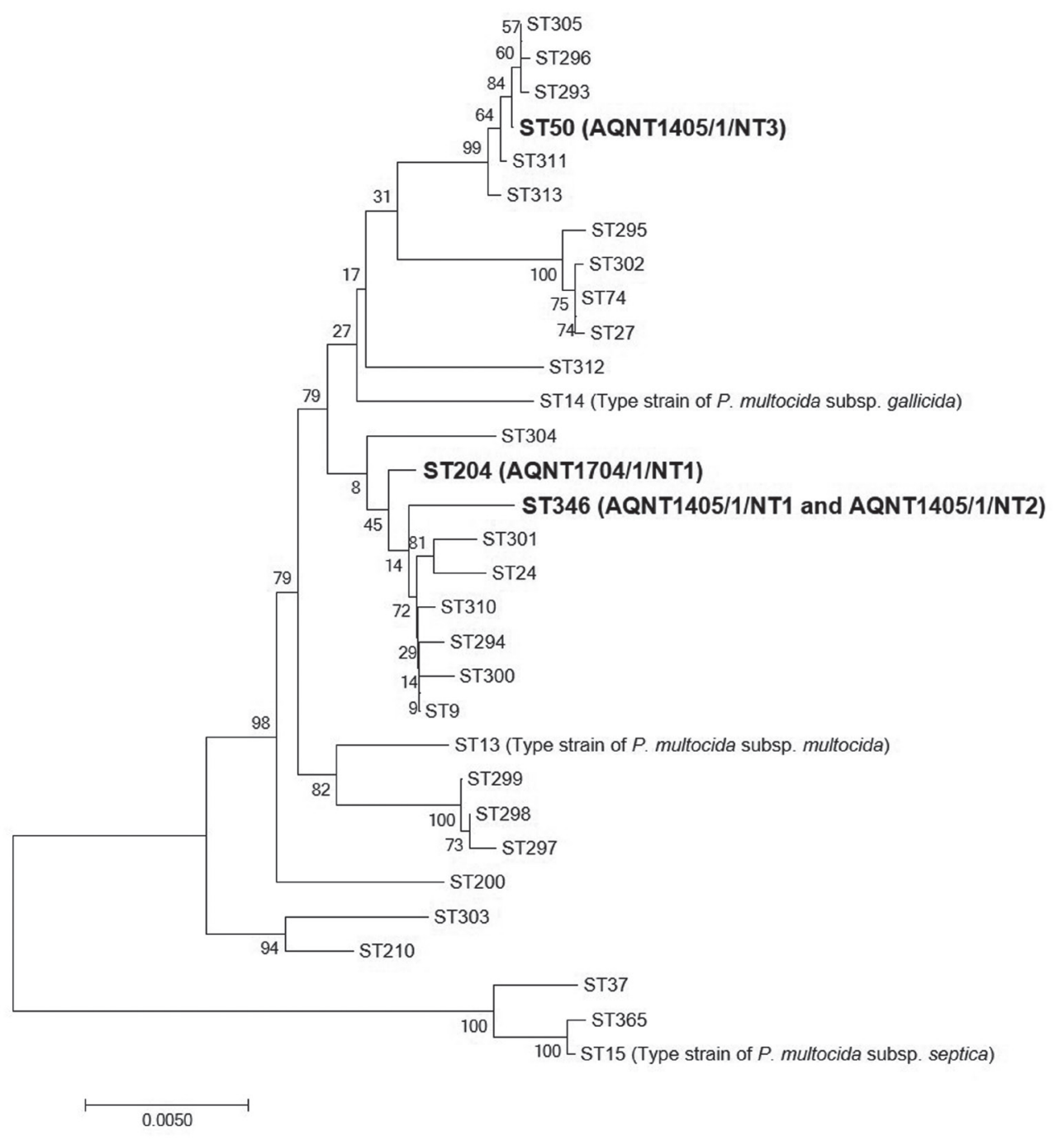

Fig. 2. Phylogenic tree of the sequence types of Pasteurella multocida strains isolated from rabbit and reference strains. Sequence types of the P. multocida strains used in this study (ST50, ST204, and ST346) are shown in bold text. The bootstrap values (1,000 replicates) [6] are shown next to the branches. Bar=the number of base differences per site. 
the database. Almost all P. multocida isolates from rabbit in the MLST database are from Europe and Oceania. To estimate the phylogenetic relationships and genetic population of $P$. multocida strains from rabbit more accurately, further epidemiological investigation is needed with more strains, especially those isolated from Asia and regions other than Europe and Oceania.

In conclusion, we have reported the immunohistochemical diagnosis of rabbit pasteurellosis. Subsequently, the isolated P. multocida serotype A: 11 was classified as ST204 using the RIRDC scheme of MLST. This is the first report using anti- $P$. multocida somatic chicken antisera in rabbit specimens, which proved useful for immunochemical identification. The present findings emphasize the need to examine the phylogenetic relationships and genetic population of $P$. multocida strains from rabbits, through further epidemiological investigation with more strains, especially those isolated in regions besides Europe and Oceania.

ACKNOWLEDGMENTS. The authors thank Mr. M. Kobayashi and Ms. M. Shimada, for histopathological assistance.

\section{REFERENCES}

1. Brogden, K. A. 1980. Physiological and serological characteristics of 48 Pasteurella multocida cultures from rabbits. J. Clin. Microbiol. 11: 646-649. [Medline]

2. Carter, G. R. 1952. The type specific capsular antigen of Pasteurella multocida. Can. J. Med. Sci. 30: 48-53. [Medline]

3. Chengappa, M. M., Myers, R. C. and Carter, G. R. 1982. Capsular and somatic types of Pasteurella multocida from rabbits. Can. J. Comp. Med. 46: 437-439. [Medline]

4. Dabo, S. M., Debey, B. M., Montelongo, M. and Confer, A. W. 1999. Genomic DNA restriction site heterogeneity in bovine Pasteurella multocida serogroup A isolates detected with an rRNA probe. J. Med. Microbiol. 48: 279-286. [Medline] [CrossRef]

5. Enright, M. C. and Spratt, B. G. 1999. Multilocus sequence typing. Trends Microbiol. 7: 482-487. [Medline] [CrossRef]

6. Felsenstein, J. 1985. Confidence limits on phylogenies: an approach using the bootstrap. Evolution 39: 783-791. [Medline] [CrossRef]

7. Flatt, R. E. and Dungworth, D. L. 1971. Enzootic pneumonia in rabbits: naturally occurring lesions in lungs of apparently healthy young rabbits. Am. J. Vet. Res. 32: 621-626. [Medline]

8. Fox, R. R., Norberg, R. F. and Myers, D. D. 1971. The relationship of Pasteurella multocida to otitis media in the domestic rabbit (Oryctolagus cuniculus). Lab. Anim. Sci. 21: 45-48. [Medline]

9. Hagen, K. W. Jr. 1958. Enzootic pasteurellosis in domestic rabbits. I. Pathology and becteriology. J. Am. Vet. Med. Assoc. 133: 77-80. [Medline]

10. Hagen, K. W. Jr. 1959. Chronic respiratory infection in the domestic rabbit. Proc. Anim. Care Panel 9: 55-60.

11. Heddleston, K. L., Gallagher, J. E. and Rebers, P. A. 1972. Fowl cholera: gel diffusion precipitin test for serotyping Pasteruella multocida from avian species. Avian Dis. 16: 925-936. [Medline] [CrossRef]

12. Jaglic, Z., Kucerova, Z., Nedbalcova, K., Hlozek, P. and Bartos, M. 2004. Identification of Pasteurella multocida Serogroup F isolates in rabbits. J. Vet. Med. B Infect. Dis. Vet. Public Health 51: 467-469. [Medline] [CrossRef]

13. Jolley, K. A. and Maiden, M. C. 2010. BIGSdb: Scalable analysis of bacterial genome variation at the population level. BMC Bioinformatics 11: 595. [Medline] [CrossRef]

14. Kumar, S., Stecher, G. and Tamura, K. 2016. MEGA7: Molecular Evolutionary Genetics Analysis version 7.0 for bigger datasets. Mol. Biol. Evol. 33: 1870-1874. [Medline] [CrossRef]

15. Lu, Y. S., Pakes, S. P. and Stefanu, C. 1983. Capsular and somatic serotypes of Pasteurella multocida isolates recovered from healthy and diseased rabbits in Texas. J. Clin. Microbiol. 18: 292-295. [Medline]

16. Nei, M. and Kumar, S. 2000. Molecular Evolution and Phylogenetics. Oxford University Press, Oxford.

17. Palócz, O., Gál, J., Clayton, P., Dinya, Z., Somogyi, Z., Juhász, C. and Csikó, G. 2014. Alternative treatment of serious and mild Pasteurella multocida infection in New Zealand White rabbits. BMC Vet. Res. 10: 276. [Medline] [CrossRef]

18. Saitou, N. and Nei, M. 1987. The neighbor-joining method: a new method for reconstructing phylogenetic trees. Mol. Biol. Evol. 4: 406-425. [Medline]

19. Stahel, A. B., Hoop, R. K., Kuhnert, P. and Korczak, B. M. 2009. Phenotypic and genetic characterization of Pasteurella multocida and related isolates from rabbits in Switzerland. J. Vet. Diagn. Invest. 21: 793-802. [Medline] [CrossRef]

20. Subaaharan, S., Blackall, L. L. and Blackall, P. J. 2010. Development of a multi-locus sequence typing scheme for avian isolates of Pasteurella multocida. Vet. Microbiol. 141: 354-361. [Medline] [CrossRef]

21. Takashima, H., Sakai, H., Yanai, T. and Masegi, T. 2001. Detection of antibodies against Pasteurella multocida using immunohistochemical staining in an outbreak of rabbit pasteurellosis. J. Vet. Med. Sci. 63: 171-174. [Medline] [CrossRef]

22. Therese, K. L., Bartell, J., Deepa, P., Mangaiyarkarasi, S., Ward, D., Dajcs, J., Madhavan, H. N. and Stroman, D. 2009. DNA sequencing by Microseq kit targeting 16S rRNA gene for species level identification of mycobacteria. Indian J. Med. Res. 129: 176-181. [Medline]

23. Townsend, K. M., Boyce, J. D., Chung, J. Y., Frost, A. J. and Adler, B. 2001. Genetic organization of Pasteurella multocida cap loci and development of a multiplex capsular PCR typing system. J. Clin. Microbiol. 39: 924-929. [Medline] [CrossRef]

24. Wagner, J., Coupland, P., Browne, H. P., Lawley, T. D., Francis, S. C. and Parkhill, J. 2016. Evaluation of PacBio sequencing for full-length bacterial 16S rRNA gene classification. BMC Microbiol. 16: 274. [Medline] [CrossRef]

25. Yoon, S. H., Ha, S. M., Kwon, S., Lim, J., Kim, Y., Seo, H. and Chun, J. 2017. Introducing EzBioCloud: a taxonomically united database of 16 S rRNA gene sequences and whole-genome assemblies. Int. J. Syst. Evol. Microbiol. 67: 1613-1617. [Medline] [CrossRef] 